\title{
Perspectives on Salutogenesis of Scholars Writing in Spanish
}

\author{
Dolors Juvinyà-Canal, Mariano Hernán, and Javier Gallego-Diéguez
}

\section{Introduction}

In the last few years, the influence of salutogenesis has been growing, both in the area of research and in the public health and health promotion strategies and policies, in Spain and in Latin America. Since Hernán and Lineros (2000) reviewed this model in Spain, the number of publications that apply Antonovsky's perspective, as well as the actions based on the health assets model, have increased.

In the last few years, this model has had an impact on the health promotion actions which have been carried out in the different scenarios. More precisely, in school health promotion, some actions oriented to promote the personal and social development of educators have been promoted, and both internal and external personal resources have been considered as an important way to reinforce general resistance resources. Proposals that promote emotional health, coexistence, resilience, and physical activity have been emphasized. Another important area that has been developed lately is the momentum of the Spanish Health Promotion Universities Network and the Latin America Health Promotion Universities Network (REUS and RIUPS, respectively, in Spanish) for setting up synergies among university members and for improving health promotion education and research. The asset maps methodology is being used in order to identify health resources. In 2013, the Alliance for Community Health was set up by many Spanish Public Health Administration entities, by the Medicine, Nursing

D. Juvinyà-Canal $(\bowtie)$

University of Girona, Girona, Spain

e-mail: dolors.juvinya@udg.edu

\section{Hernán}

Andalusian School of Public Health, Granada, Spain

e-mail: mariano.hernan.easp@juntadeandalucia.es

J. Gallego-Diéguez

Aragon Government Directorate of Public Health, Zaragoza, Spain

e-mail: jgallego@aragon.es and Public Health Scientific Societies and by the Public Health Universities and Education Centers, with the aim to promote community health from primary care health services.

The analysis of health social determinants and health equity has generated a large debate and has led to the development of different strategies and plans to reduce health social inequalities. The salutogenesis and health asset model is extremely useful when it is included in policies and programs whose aim is to achieve health equity.

\section{Review of the Scientific Literature on Salutogenesis in Spanish}

This literature has been searched from 2000 on in the Pubmed, Cinhal, Medline, Cochrane, Redalyc, and Scopus databases, by entering the following keywords: salutogenesis, sense of coherence, salutogenic, salutogenic approach, health assets, and SOC.

The selected papers were classified by their year of publication, publication type, and these topics:

- Sense of coherence

- Salutogenic policies

- Salutogenesis approach.

- Health assets

From the year 2000 to this writing, the salutogenesis literature in Spanish includes 58 articles, five books, and eight doctoral dissertations, produced in Spain, Portugal, and countries of Latin America. Tables 47.1, 47.2, 47.3, and 47.4 list journal articles in the ascending order of year of publication, with citation information in Spanish. The last column in each Table indicates in English the study group or groups that are in focus in each article. 
Table 47.1 Sense of coherence-journal articles

\begin{tabular}{|c|c|c|c|c|c|}
\hline Date & Authors & Article title & Journal title & $\begin{array}{l}\text { Volume (issue): } \\
\text { pages }\end{array}$ & Study group(s) \\
\hline 2004 & $\begin{array}{l}\text { Chacón Roger, M.; Jorge } \\
\text { Grau, A. }\end{array}$ & $\begin{array}{l}\text { Burnout y variables personales moduladoras } \\
\text { en enfermeros que trabajan en hospitales } \\
\text { oncológicos }\end{array}$ & $\begin{array}{l}\text { Psicología y } \\
\text { Salud de la } \\
\text { Universidad } \\
\text { Veracruzana }\end{array}$ & 14(1):67-78 & Nurses \\
\hline 2007 & Segura, A. & La prevención de la dependencia & $\begin{array}{l}\text { Revista española } \\
\text { de geriatría y } \\
\text { gerontología }\end{array}$ & $42(2): 7-11$ & Dependents \\
\hline 2007 & Dejo Vásquez, M. & $\begin{array}{l}\text { Sentido de coherencia, afrontamiento y } \\
\text { sobrecarga en cuidadores familiares de } \\
\text { ancianos con enfermedad crónica }\end{array}$ & $\begin{array}{l}\text { Avances en } \\
\text { psicología } \\
\text { latino-Americana }\end{array}$ & $25(1): 64-71$ & $\begin{array}{l}\text { Elderly } \\
\text { relatives with } \\
\text { chronic illness }\end{array}$ \\
\hline 2007 & $\begin{array}{l}\text { Virués-Ortega, J.; Martínez, } \\
\text { P.; del Barrio, J.L.; Lozano, L. }\end{array}$ & $\begin{array}{l}\text { Validación transcultural de la Escala de } \\
\text { Sentido de Coherencia de Antonovsky } \\
\text { (OLQ-13) en ancianos mayores de } 70 \text { años }\end{array}$ & Medicina clínica & 128(13):486-492 & Elderly \\
\hline 2008 & $\begin{array}{l}\text { Besteiro, J.; Álvarez, M., } \\
\text { Lemos, S.; Muñiz, J.; Costas, } \\
\text { C.; Weruaga, A. }\end{array}$ & $\begin{array}{l}\text { Dimensiones de personalidad, sentido de } \\
\text { coherencia y salud percibida en pacientes con } \\
\text { un síndrome fibromiálgico }\end{array}$ & $\begin{array}{l}\text { International } \\
\text { Journal of } \\
\text { Clinical and } \\
\text { Health } \\
\text { Psychology } \\
\end{array}$ & $8(2): 411-427$ & $\begin{array}{l}\text { Patients with } \\
\text { fibromyalgia } \\
\text { syndrome }\end{array}$ \\
\hline 2008 & 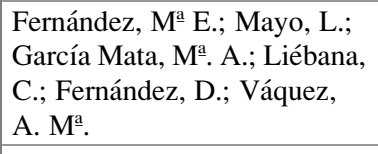 & $\begin{array}{l}\text { Sentido de coherencia y salud percibida en } \\
\text { alumnos universitarios de ciencias de la salud }\end{array}$ & $\begin{array}{l}\text { Revista Española } \\
\text { de Sanidad } \\
\text { Penitenciaria }\end{array}$ & $5(3): 1-5$ & $\begin{array}{l}\text { University } \\
\text { students of } \\
\text { health sciences }\end{array}$ \\
\hline 2008 & Ureña Bonilla, P. & $\begin{array}{l}\text { Calidad de vida, sentido de coherencia y } \\
\text { niveles de sedentarismo en académicos y } \\
\text { administrativos del campus presbítero } \\
\text { Benjamín Núñez, U.N.A. }\end{array}$ & $\begin{array}{l}\text { Revista } M H \\
\text { Salud }\end{array}$ & $5(2): 1-15$ & $\begin{array}{l}\text { Academic and } \\
\text { administrative } \\
\text { staff }\end{array}$ \\
\hline 2009 & $\begin{array}{l}\text { Ureña Bonilla, P.; Castro } \\
\text { Sancho, C. }\end{array}$ & $\begin{array}{l}\text { Calidad de vida, sentido de coherencia y } \\
\text { satisfacción laboral en profesores(as) de } \\
\text { colegios técnicos en la Dirección Regional de } \\
\text { Heredia }\end{array}$ & Educare & 13(1):71-87 & $\begin{array}{l}\text { Teachers of } \\
\text { technical } \\
\text { colleges }\end{array}$ \\
\hline 2011 & $\begin{array}{l}\text { Robledo-Martínez,R.; } \\
\text { Agudelo-Calderón, C. }\end{array}$ & $\begin{array}{l}\text { Aproximación a la construcción teórica de la } \\
\text { promoción de la salud }\end{array}$ & $\begin{array}{l}\text { Revista de Salud } \\
\text { Pública }\end{array}$ & 13(6):1031-1050 & \\
\hline 2011 & $\begin{array}{l}\text { Rodríguez Costa Schmidt, D.; } \\
\text { Aparecida Spadoti Dantas, R. }\end{array}$ & $\begin{array}{l}\text { Análisis de validez y confiabilidad de la } \\
\text { versión adaptada para el portugués del } \\
\text { Cuestionario de Sentido de Coherencia de } \\
\text { Antonovsky entre profesionales de } \\
\text { enfermería }\end{array}$ & $\begin{array}{l}\text { Revista Latino- } \\
\text { Americana de } \\
\text { Enfermagem }\end{array}$ & $19(1): 1-8$ & Nurses \\
\hline 2012 & $\begin{array}{l}\text { Malagón, M. C.; Juvinyà,D.;; } \\
\text { Bonmatí, A.; Fernández, R.; } \\
\text { Bosch, C.; Bertrna,C.; Suñer, } \\
\text { R. }\end{array}$ & $\begin{array}{l}\text { El sentido de coherència de las enferemeras y } \\
\text { validación del cuestionario SOC-13 }\end{array}$ & $\begin{array}{l}\text { Metas de } \\
\text { Enfermería }\end{array}$ & $15(9): 27-31$ & Nurses \\
\hline 2012 & $\begin{array}{l}\text { Malagón, M. C.; Fuentes, C.; } \\
\text { Suñer, R; Bonmatí, A.; } \\
\text { Fernández, R.; Bosch, C. }\end{array}$ & $\begin{array}{l}\text { El sentido de coherencia en el colectivo } \\
\text { enfermero }\end{array}$ & $\begin{array}{l}\text { Enfermería } \\
\text { Clínica }\end{array}$ & $22(4): 214-218$ & Patients \\
\hline 2013 & $\begin{array}{l}\text { Paredes, J.; Agulló, J.; Vera, } \\
\text { E.; Hernán, M. }\end{array}$ & $\begin{array}{l}\text { Sentido de coherencia y activos para la Salud } \\
\text { en jóvenes internos en centros de Menores }\end{array}$ & $\begin{array}{l}\text { Revista Española } \\
\text { de Sanidad }\end{array}$ & $15: 87-97$ & $\begin{array}{l}\text { Young } \\
\text { inmates }\end{array}$ \\
\hline 2013 & $\begin{array}{l}\text { García-Moya, I.; Rivera, F.; } \\
\text { Moreno, C.; López, A. }\end{array}$ & $\begin{array}{l}\text { Calidad de la relación entre los progenitores y } \\
\text { sentido de coherencia en sus hijos } \\
\text { adolescentes. El efecto de mediación de la } \\
\text { satisfacción familiar }\end{array}$ & $\begin{array}{l}\text { Anales de } \\
\text { psicología }\end{array}$ & $29(2): 482-490$ & Teens \\
\hline
\end{tabular}

Besides the salutogenesis literature in journals, as listed in Table 47.5, several noteworthy books have been published. The first one was published in 2000, and the other four, were published after 10 years, one book on 2010 and three books on 2011. The last column of Table 47.5 indicates the issue covered in the book.
Finally, eight doctoral dissertations have been identified. Also, in Table 47.6, the last column indicates the subject of the doctoral dissertation, and five of the eight focus on population groups. 
Table 47.2 Salutogenic policies-journal articles

\begin{tabular}{|c|c|c|c|c|c|}
\hline Date & Authors & Article title & Journal title & $\begin{array}{l}\text { Volume (issue) } \\
\text { page }\end{array}$ & $\begin{array}{l}\text { Study } \\
\text { group }\end{array}$ \\
\hline 2008 & Sanabria Ferrand, P.A. & $\begin{array}{l}\text { Reseña de "Psicología de la Salud: Temas Actuales } \\
\text { de Investigación en Latinoamérica" de L. Flórez } \\
\text { Alarcón, M.M. Botero y B. Moreno Jiménez (eds.) }\end{array}$ & $\begin{array}{l}\text { Inter-American } \\
\text { Journal of } \\
\text { Psychology }\end{array}$ & 42(1):181-182 & \\
\hline 2008 & Segura, A. & $\begin{array}{l}\text { Políticas de salud (actuaciones poblacionales) en los } \\
\text { servicios asistenciales. Informe SESPAS } 2008\end{array}$ & Gaceta Sanitaria & $22(1): 104-110$ & $\begin{array}{l}\text { Care } \\
\text { services }\end{array}$ \\
\hline 2009 & $\begin{array}{l}\text { Omar, A.; Paris, L.; Aguilar de } \\
\text { Souza, M.; Almeida da Silva, } \\
\text { S.E.; del Pino Peña, R. }\end{array}$ & $\begin{array}{l}\text { Validación del inventario de bienestar subjetivo con } \\
\text { muestras de jóvenes y adolescentes argentinos, } \\
\text { brasileros y mexicanos }\end{array}$ & Suma Psicológica & $16(2): 69-84$ & Teens \\
\hline 2010 & De Lellis, M. & Psicología y políticas públicas saludables & $\begin{array}{l}\text { Revista } \\
\text { Latinoamericana } \\
\text { de Ciencia } \\
\text { Psicológica }\end{array}$ & $2(2): 102-106$ & \\
\hline 2011 & $\begin{array}{l}\text { Álvarez-Dardet, C.; Ruiz } \\
\text { Cantero, Mํ․ T. }\end{array}$ & $\begin{array}{l}\text { Patrimonio de salud ¿Son posibles las políticas } \\
\text { salutogénicas? }\end{array}$ & $\begin{array}{l}\text { Revista Española } \\
\text { de Salud Pública }\end{array}$ & $85(2): 123-127$ & \\
\hline 2012 & $\begin{array}{l}\text { Agost Felip, Ma R.; Martín } \\
\text { Alfonso, L. }\end{array}$ & $\begin{array}{l}\text { Acercamiento al papel de los procesos de exclusión } \\
\text { social y su relación con la salud }\end{array}$ & $\begin{array}{l}\text { Revista Cubana } \\
\text { de Salud Pública }\end{array}$ & $38(1): 126-140$ & \\
\hline
\end{tabular}

\section{Spanish Salutogenesis Group}

The Spanish Salutogenesis Group was set up on July 12, 2012, stimulated by a proposal of Professor Bengt Lindström when he visited the University of Girona (Spain) for the presentation of the book "The Salutogenic Hitchhiker," which had been translated into Spanish by the Health Promotion Chair at the University of Girona. This group was set up by the authors of this chapter with the aim to create a meeting point, a resource for Spanish-speaking professionals who are working or wish to work from the salutogenic approach, as well as to promote the work that is being carried out in Spanish speaking countries and to build a bridge with the international group. The group's objectives are to:

- Expand the network of professionals working in salutogenesis in Spanish.

- Promote and exchange experiences and knowledge on salutogenesis.

- Promote salutogenesis research and development within our contexts.

- Stimulate the implementation of the salutogenic approach in intervention, education, and research.

In order to develop a strategy that fosters the salutogenic approach, based on assets for public health, the Salutogenesis Spanish Group proposes the ten principles below:

1. The contributions of sociology, psychology, and human learning theories to medicine and health sciences have generated a salutogenic approach to public health. This new perspective complements and, somehow, also opposes the more traditional perspective of the biomedical sciences, which focuses on the recovery of health deficits. Salutogenesis is re-oriented towards people's well-being.

2. The salutogenic approach emphasizes the assets that generate health and also examines the elements that make it possible for people to understand, manage, and find a sense to their life. These elements focus on the individual and group resources available-the so-called general resistance resources - that help people to understand their own life, the effects of the structural inequalities and therefore become stronger to overcome them. This perspective and its health paradigms are especially necessary now, in a context of global crisis and deep economic changes that can impact people and foster social inequalities which, in turn, have an impact on health.

3. The existing knowledge should be rebuilt and new knowledge should be gathered, in order to provide legislators, politicians, managers, promotion specialists, and professionals in the health, well-being, and education sectors with positive approaches for health, wellbeing, and human development. The salutogenic approach should be included in public health regulations, plans, and programs.

4. Promoting an approach to public health based on the vital life of people could make it easier to understand and to manage health assets in each phase of life. If health is included in all public policies, we will be able to understand people and communities in their contexts and in the different phases of life, and clarify the changes required in policies for better health and wellbeing. 
Table 47.3 Salutogenic approach-journals articles

\begin{tabular}{|c|c|c|c|c|c|}
\hline Date & Authors & Article title & Journal title & $\begin{array}{l}\text { Volume (issue) } \\
\text { page }\end{array}$ & $\begin{array}{l}\text { Study } \\
\text { group }\end{array}$ \\
\hline 2001 & $\begin{array}{l}\text { Hernán, M.; Ramos, M.; } \\
\text { Fernandez, A. }\end{array}$ & $\begin{array}{l}\text { Revisión de los trabajos publicados sobre } \\
\text { promoción de la salud en jóvenes españoles }\end{array}$ & $\begin{array}{l}\text { Revista Española } \\
\text { de Salud Pública }\end{array}$ & $75(6): 491-503$ & Teens \\
\hline 2002 & Franco, G. A. & Editorial: Los temas de la salud pública & $\begin{array}{l}\text { Revista Facultad } \\
\text { Nacional de Salud } \\
\text { Pública }\end{array}$ & $20(1): 1-2$ & \\
\hline 2002 & $\begin{array}{l}\text { García Martín, M. A.; Hombrados } \\
\text { Mendieta, Mª I. }\end{array}$ & $\begin{array}{l}\text { Control percibido y bienestar subjetivo: un } \\
\text { análisis de la literatura gerontológica }\end{array}$ & $\begin{array}{l}\text { Revista } \\
\text { multidisciplinar } \\
\text { de gerontología }\end{array}$ & $12(2): 90-102$ & Elderly \\
\hline 2009 & Londoño Pérez, C. & $\begin{array}{l}\text { Optimismo y salud positiva como predictores } \\
\text { de la adaptación a la vida universitaria }\end{array}$ & $\begin{array}{l}\text { Acta Colombiana } \\
\text { de Psicología }\end{array}$ & $12(1): 95$ & $\begin{array}{l}\text { College } \\
\text { students }\end{array}$ \\
\hline 2009 & $\begin{array}{l}\text { Sánchez, D.; Lineros, C.; Hernán } \\
\text { M. }\end{array}$ & $\begin{array}{l}\text { Potenciales activos para la salud de la juventud } \\
\text { en Nicaragua }\end{array}$ & Gaceta Sanitaria & $20: 303$ & $\begin{array}{l}\text { Young } \\
\text { people }\end{array}$ \\
\hline 2009 & $\begin{array}{l}\text { Pérez, M.; Jiménez, J.; García, J.; } \\
\text { Hernán, M. }\end{array}$ & $\begin{array}{l}\text { Salud en internet para adolescentes y los } \\
\text { jóvenes }\end{array}$ & Gaceta Sanitaria & 13:138 & $\begin{array}{l}\text { Teens } \\
\text { and } \\
\text { young } \\
\text { people }\end{array}$ \\
\hline 2011 & $\begin{array}{l}\text { Rivera de los Santos, F.; Ramos } \\
\text { Valverde, P.; Moreno Rodríguez, } \\
\text { C.; Hernán García, M. }\end{array}$ & $\begin{array}{l}\text { Análisis del modelo salutogénico en España: } \\
\text { aplicación en salud pública e implicaciones } \\
\text { para el modelo de activos en salud }\end{array}$ & $\begin{array}{l}\text { Revista Española } \\
\text { de Salud Pública }\end{array}$ & $85(2): 129-139$ & \\
\hline 2011 & Morelato, G. & $\begin{array}{l}\text { Maltrato infantil y desarrollo: hacia una } \\
\text { revisión de los factores de resiliencia }\end{array}$ & $\begin{array}{l}\text { Pensamiento } \\
\text { Psicológico }\end{array}$ & $9(17): 83-96$ & Infants \\
\hline 2011 & Juárez, F. & $\begin{array}{l}\text { El concepto de salud: Una explicación sobre su } \\
\text { unicidad, multiplicidad y los modelos de salud }\end{array}$ & $\begin{array}{l}\text { International } \\
\text { Journal of } \\
\text { Psychological } \\
\text { Research }\end{array}$ & $4(1): 70$ & \\
\hline 2012 & $\begin{array}{l}\text { Santana Bravo, F.; Martin Castillo, } \\
\text { D.; Camuñez Gómez, MD.; Bueno } \\
\text { Balboteo, JM. }\end{array}$ & $\begin{array}{l}\text { Estudio sobre hábitos alimenticios y actividad } \\
\text { física en nuestros adolescentes }\end{array}$ & $\begin{array}{l}\text { Nutrición } \\
\text { Hospitalaria }\end{array}$ & $27(3): 53-75$ & Teens \\
\hline 2012 & Cofiño, R.; Pasarín, M I.; Segura, A. & $\begin{array}{l}\text { ¿Cómo abordar la dimensión colectiva de la } \\
\text { salud de las personas? Informe SESPAS } 2012\end{array}$ & Gaceta Sanitaria & $26(1): 88-93$ & \\
\hline 2012 & Oliveira, C.; Costa, A. L. & $\begin{array}{l}\text { Viver o estado terminal de um familiar: leitura } \\
\text { salutogénica de resultados de um estudo de caso }\end{array}$ & $\begin{array}{l}\text { Revista } \\
\text { electrónica online } \\
\text { (Scielo) }\end{array}$ & 21(3):698-709 & \\
\hline 2012 & Mariñelarena-Dondena, L. & $\begin{array}{l}\text { Recepción y desarrollo de la psicología positiva } \\
\text { en la Universidad de Buenos Aires } \\
\text { (1998-2008) }\end{array}$ & $\begin{array}{l}\text { Revista } \\
\text { Latinoamericana } \\
\text { de Ciencia } \\
\text { Psicológica }\end{array}$ & $4(2): 76-83$ & \\
\hline 2012 & Saforcada, E. & $\begin{array}{l}\text { Psicología sanitaria: historia, fundamentos y } \\
\text { perspectivas }\end{array}$ & $\begin{array}{l}\text { Revista } \\
\text { Latinoamericana } \\
\text { de Ciencia } \\
\text { Psicológica }\end{array}$ & $4(2): 120-130$ & \\
\hline 2012 & $\begin{array}{l}\text { Ortega-Calvo, M.; Santos, J. M.; } \\
\text { Lapetra, J. }\end{array}$ & La animación científica en atención primaria & $\begin{array}{l}\text { Atención } \\
\text { Primaria }\end{array}$ & 44(9):549-554 & \\
\hline 2012 & $\begin{array}{l}\text { Bakker, Arnold B.; Rodríguez } \\
\text { Muñoz, A.; Derks, D. }\end{array}$ & $\begin{array}{l}\text { La emergencia de la psicología de la salud } \\
\text { ocupacional positiva }\end{array}$ & Psicothema & $24(1): 66-72$ & \\
\hline 2012 & $\begin{array}{l}\text { Bakker, Arnold B.; Rodríguez } \\
\text { Muñoz, A. }\end{array}$ & $\begin{array}{l}\text { Introducción a la psicología de la salud } \\
\text { ocupacional positiva }\end{array}$ & Psicothema & $24(1): 62-65$ & \\
\hline 2012 & Thielmann, K.; Illnait Ferrer, J. & La crisis y la salud. ¿La salud en crisis? & $\begin{array}{l}\text { Revista Cubana } \\
\text { de Salud Pública }\end{array}$ & $38(2): 278-285$ & \\
\hline 2013 & Juvinyà, D. & $\begin{array}{l}\text { Salutogénesis, nuevas perspectivas para } \\
\text { promover la salud }\end{array}$ & $\begin{array}{l}\text { Enfermería } \\
\text { Clínica }\end{array}$ & $23(3): 87-88$ & \\
\hline
\end{tabular}


Table 47.4 Health assets-journal articles

\begin{tabular}{|c|c|c|c|c|c|}
\hline Date & Authors & Article title & Journal title & $\begin{array}{l}\text { Volume (issue) } \\
\text { page }\end{array}$ & $\begin{array}{l}\text { Study } \\
\text { group }\end{array}$ \\
\hline 2009 & Botello, B.; Hernán,M. & $\begin{array}{l}\text { Opiniones de los jóvenes sobre la salud mental } \\
\text { en Huelva según el modelo de activos. }\end{array}$ & $\begin{array}{l}\text { Gaceta } \\
\text { Sanitaria }\end{array}$ & $33(96)$ & $\begin{array}{l}\text { Young } \\
\text { people }\end{array}$ \\
\hline 2010 & Hernán, M.; Lineros, C. & $\begin{array}{l}\text { Los activos para la salud. Promoción de la } \\
\text { salud en contextos personales, familiares y } \\
\text { sociales. }\end{array}$ & $\begin{array}{l}\text { Rev. } \\
\text { FUNDESFAM }\end{array}$ & $2(2)$ & \\
\hline 2011 & $\begin{array}{l}\text { Rivera de los Santos F.; Ramos P.; } \\
\text { Moreno C.; Hernán M. }\end{array}$ & $\begin{array}{l}\text { Análisis del modelo salotogénico en España: } \\
\text { aplicación en salud pública e implicacions } \\
\text { para el modelo de activos en salud }\end{array}$ & $\begin{array}{l}\text { Revista } \\
\text { Española de } \\
\text { Salud Pública }\end{array}$ & $85(2): 129-139$ & \\
\hline 2012 & $\begin{array}{l}\text { Ramos-Morcilloa, A.J.; Fernández- } \\
\text { Salazar, S. }\end{array}$ & $\begin{array}{l}\text { Cuidando a un preescolar desde el modelo de } \\
\text { activos en salud: caso clínico }\end{array}$ & $\begin{array}{l}\text { Enfermería } \\
\text { Clínica }\end{array}$ & $22(3): 166-169$ & Preschool \\
\hline 2012 & Hernán, M. & $\begin{array}{l}\text { Activos para la Salud y Salutogénesis; } \\
\text { emergentes en Salud Pública }\end{array}$ & bepSALUT & 1 & \\
\hline 2013 & Gómez-Acosta, C. A.; Londoño Pérez, C. & $\begin{array}{l}\text { Modelo predictor del consumo responsable de } \\
\text { alcohol y el comportamiento típicamente no } \\
\text { violento en adolescentes }\end{array}$ & $\begin{array}{l}\text { Health and } \\
\text { addictions: } \\
\text { salud y drogas }\end{array}$ & $13(1): 23-34$ & Teens \\
\hline 2013 & $\begin{array}{l}\text { Botello, B.; Palacio, S.; García, M.; } \\
\text { Margolles, M.; Fernández, F.; Hernán, } \\
\text { M.; Nieto, J.; Cofiño, R. }\end{array}$ & $\begin{array}{l}\text { Metodología para el mapeo de activos de salud } \\
\text { en una comunidad }\end{array}$ & $\begin{array}{l}\text { Gaceta } \\
\text { Sanitaria }\end{array}$ & $27(2): 180-183$ & \\
\hline 2012 & Cassaretto, M.; Martínez Uribe, P. & $\begin{array}{l}\text { Razones para vivir en jóvenes adultos: } \\
\text { validación del RFL-YA }\end{array}$ & $\begin{array}{l}\text { Revista de } \\
\text { Psicología }\end{array}$ & 30(1):169-188 & $\begin{array}{l}\text { Young } \\
\text { people }\end{array}$ \\
\hline 2012 & $\begin{array}{l}\text { Fernández, Me R.; Thielmann, K.; } \\
\text { Bormey Quiñones, MB. }\end{array}$ & $\begin{array}{l}\text { Determinantes individuales y sociales de salud } \\
\text { en la medicina familiar }\end{array}$ & $\begin{array}{l}\text { Revista } \\
\text { cubana de } \\
\text { salud pública }\end{array}$ & $38(3): 484$ & \\
\hline 2012 & Ruiz-Azarola, A.; Perestelo-Pérez, L. & $\begin{array}{l}\text { Participación ciudadana en salud: formación y } \\
\text { toma de decisiones compartida. Informe } \\
\text { SESPAS } 2012\end{array}$ & $\begin{array}{l}\text { Gaceta } \\
\text { Sanitaria }\end{array}$ & $26: 158-161$ & \\
\hline 2012 & Agost Felip, M르. R.; Martín Alfonso, L. & $\begin{array}{l}\text { Acercamiento al papel de los procesos de } \\
\text { exclusión social y su relación con la salud }\end{array}$ & $\begin{array}{l}\text { Revista } \\
\text { Cubana de } \\
\text { Salud Pública } \\
\end{array}$ & $38(1): 126-140$ & \\
\hline 2013 & Pérez Jarauta, M.J.; Echauri Ozcoidi, M. & $\begin{array}{l}\text { Educación versus coerción. Una apuesta } \\
\text { decidida por la educación para la salud }\end{array}$ & $\begin{array}{l}\text { Gaceta } \\
\text { Sanitaria }\end{array}$ & $27(1): 72-74$ & \\
\hline 2013 & Morgan, A.; Hernán, M. & $\begin{array}{l}\text { Promoción de la salud y el bienestar a través } \\
\text { del modelo de activos }\end{array}$ & $\begin{array}{l}\text { Revista } \\
\text { Española de } \\
\text { Sanidad }\end{array}$ & $15(3): 78-86$ & \\
\hline
\end{tabular}

Table 47.5 Salutogenesis books

\begin{tabular}{|c|c|c|c|c|}
\hline Date & Authors & Title & $\begin{array}{l}\text { Publishing } \\
\text { Company }\end{array}$ & Issue \\
\hline 2000 & Grün, Anselm & $\begin{array}{l}\text { ¿Qué enferma y qué sana a los } \\
\text { hombres? }\end{array}$ & Verbo Divino & $\begin{array}{l}\text { Factors that } \\
\text { make people ill } \\
\text { or better }\end{array}$ \\
\hline 2010 & $\begin{array}{l}\text { Hernán García, M.; } \\
\text { Lúis Mena Jiménez, Á.; Morgan, A. }\end{array}$ & $\begin{array}{l}\text { Formación en salutogénesis y activos } \\
\text { para la salud }\end{array}$ & $\begin{array}{l}\text { Escuela } \\
\text { Andaluza de } \\
\text { Salud Pública }\end{array}$ & $\begin{array}{l}\text { Assets for } \\
\text { health }\end{array}$ \\
\hline 2011 & $\begin{array}{l}\text { Hernán García, Mariano; Lúis Mena Jiménez, Ángel; } \\
\text { Lineros González, Carmen; Botello Díaz, Blanca; } \\
\text { Cubillana de la Cruz, Pablo García; Huertas Povedano, } \\
\text { Ángeles }\end{array}$ & $\begin{array}{l}\text { Activos para la salud y promoción de } \\
\text { la salud mental }\end{array}$ & $\begin{array}{l}\text { Escuela } \\
\text { Andaluza de } \\
\text { Salud Pública }\end{array}$ & $\begin{array}{l}\text { Assets for } \\
\text { health }\end{array}$ \\
\hline 2011 & Ley C.; Rato M. & $\begin{array}{l}\text { Deporte, juego y técnicas } \\
\text { participativas como herramientas } \\
\text { terapéuticas }\end{array}$ & Lambert Acad. & $\begin{array}{l}\text { Therapeutic } \\
\text { value of certain } \\
\text { techniques }\end{array}$ \\
\hline 2011 & Lindström, B.; Eriksson, M./Traducción: Dolors Juvinyà & $\begin{array}{l}\text { Guía del autoestopista salutogénico : } \\
\text { camino salutogénico hacia la } \\
\text { promoción de la salud }\end{array}$ & $\begin{array}{l}\text { Documenta } \\
\text { Universitaria }\end{array}$ & $\begin{array}{l}\text { Translation into } \\
\text { Spanish of a } \\
\text { guide }\end{array}$ \\
\hline
\end{tabular}


Table 47.6 Salutogenesis-doctoral dissertations

\begin{tabular}{|c|c|c|c|c|}
\hline Date & Author & Title & University & Subject \\
\hline 2006 & Carrondo, E. & $\begin{array}{l}\text { Formada profissional de enfermeiros e desenvolvimento da criança: } \\
\text { contributo para um perfil centrado no paradigma salutogénico; Nurses' } \\
\text { professional training and child development: contribution to a profile } \\
\text { centered on the salutogenic paradigma }\end{array}$ & $\begin{array}{l}\text { Universidade do } \\
\text { Minho }\end{array}$ & $\begin{array}{l}\text { Training of } \\
\text { professionals }\end{array}$ \\
\hline 2009 & Ley, C. & $\begin{array}{l}\text { Acción psicosocial a través del movimiento, juego y deporte en contextos } \\
\text { de violencia y de conflicto. Investigación sobre la adecuación sociocultural } \\
\text { de la 'terapia a través del deporte' y evaluación de un programa con } \\
\text { mujeres en Guatemala }\end{array}$ & $\begin{array}{l}\text { Universidad } \\
\text { Politécnica de } \\
\text { Madrid }\end{array}$ & $\begin{array}{l}\text { Psychosocial action } \\
\text { through sport }\end{array}$ \\
\hline 2010 & $\begin{array}{l}\text { Fernández, } \\
\text { M. E. }\end{array}$ & $\begin{array}{l}\text { Estrés percibido, estrategias de afrontamiento y sentido de coherencia en } \\
\text { estudiantes de enfermería: su asociación con salud psicológica y } \\
\text { estabilidad emocional }\end{array}$ & $\begin{array}{l}\text { Universidad de } \\
\text { León }\end{array}$ & Population groups \\
\hline 2011 & Ruiz, D. & $\begin{array}{l}\text { Calidad de vida del alumnado del aula de mayores de la universidad de } \\
\text { málaga y utilización de los servicios de salud }\end{array}$ & $\begin{array}{l}\text { Universidad de } \\
\text { Málaga }\end{array}$ & Population groups \\
\hline 2012 & Bennasar, M. & $\begin{array}{l}\text { Estilos de vida y salud en estudiantes universitarios: la universidad como } \\
\text { entorno promotor de la salud }\end{array}$ & $\begin{array}{l}\text { Universidad de les } \\
\text { Illes Balears }\end{array}$ & Population groups \\
\hline 2012 & $\begin{array}{l}\text { Rivera de los } \\
\text { Santos, F. }\end{array}$ & $\begin{array}{l}\text { Salutogénesis y sentido de coherencia: un estudio psicométrico de la escala } \\
\text { SOC en adolescentes españoles }\end{array}$ & $\begin{array}{l}\text { Universidad de } \\
\text { Sevilla }\end{array}$ & Population groups \\
\hline 2013 & Bustamante, E. & $\begin{array}{l}\text { La comunicación interna y la promoción de la salud estudio de caso en } \\
\text { Madrid salud }\end{array}$ & $\begin{array}{l}\text { Universidad } \\
\text { Autónoma de } \\
\text { Barcelona }\end{array}$ & $\begin{array}{l}\text { Communication } \\
\text { and health } \\
\text { promotion }\end{array}$ \\
\hline 2013 & Carrillo, J. & $\begin{array}{l}\text { Promoción de la salud de los empleados públicos de la Región de Murcia: } \\
\text { prevención del estrés a través del Taiji Quan y Qigong }\end{array}$ & $\begin{array}{l}\text { Universidad de } \\
\text { Murcia }\end{array}$ & Population groups \\
\hline
\end{tabular}

5. Professionals should be involved with communities in all issues related to the health development process, enabling them to use all the capacities of people in a place and social context. By being aware of all we can do for our health and of what should be socially changed-and by taking the necessary steps required —we will illuminate the opportunities available to be healthy.

6. It is important to learn more about the relationship between health and the sense of coherence, which allows people to understand, manage, and find a sense to what happens along their life, and also to know how this impacts on their personal quality of life and on that of their community. An asset for health could be defined as any element or resource that reinforces the capacity of individuals, communities, and populations, so that they keep their health, well-being, and sense of coherence.

7. We should foster and improve the evidence that allows us to further understand the elements that make people and communities live a healthier life. This could help us to emphasize the health determinant analysis models that aim at understanding which is the origin of health and its correlation with quality of life. We should identify the key elements or resources that contribute to health and well-being in childhood and youth-which are crucial phases in life to learn in a healthy way-and also in other stages in life.
8. In order to develop purposes for the strategy that fosters the salutogenic approach, it is very important to apply a multidisciplinary approach and to complement experimental studies with narration, epidemiology, ethnography, sociology, and biomedical sciences.

9. A key element consists of identifying experiences, types of strategies, initiatives, and ways to work that more efficiently lead to the promotion of capacities and abilities in individuals and communities, so that health is maintained, promoted, and recovered.

10. It is absolutely necessary to work so that projects and actions include some indicators that lead to the evaluation of programs based on positive models. We should improve the ability to understand not only the things that work and their results, but also how things work in different contexts-because the social context of people helps to identify priorities and, thus, promote the elements which generate health and reduce the stress created by unfair inequalities, as well as all the elements that could help to design policies that allow people to evolve towards health objectives in the medium and long term.

The website of the Spanish Salutogenesis Group disseminates information about the Group, news related to it, resources, projects, and links. 
Open Access This chapter is distributed under the terms of the Creative Commons Attribution-Noncommercial 2.5 License (http:// creativecommons.org/licenses/by-nc/2.5/) which permits any noncommercial use, distribution, and reproduction in any medium, provided the original author(s) and source are credited.
The images or other third party material in this chapter are included in the work's Creative Commons license, unless indicated otherwise in the credit line; if such material is not included in the work's Creative Commons license and the respective action is not permitted by statutory regulation, users will need to obtain permission from the license holder to duplicate, adapt or reproduce the material. 